\title{
Erratum: Refining the boundaries of the classical de Sitter landscape
}

\author{
David Andriot $^{a, b}$ and Johan Blåbäck ${ }^{c}$ \\ ${ }^{a}$ Max-Planck-Institut für Gravitationsphysik, Albert-Einstein-Institut, \\ Am Mühlenberg 1, 14467 Potsdam-Golm, Germany \\ ${ }^{b}$ Institut für Mathematik, Humboldt-Universität zu Berlin, IRIS-Adlershof, \\ Zum Großen Windkanal 6, 12489 Berlin, Germany \\ ${ }^{c}$ Institut de Physique Théorique, Université Paris Saclay, CEA, CNRS, \\ F-91191 Gif sur Yvette, France \\ E-mail: david.andriot@aei.mpg.de, johan.blaback@cea.fr
}

ERRATUM TO: JHEP03(2017)102

ArXiv ePrint: 1609.00385

\section{Summary and consequences}

In the trace of the Einstein equation along internal parallel flat directions, namely equations (4.14) and (4.15), a few terms have been missed. As a consequence the corrected equations will have additional terms which depend on specific components of fluxes, such as $\left|H^{(2)}\right|^{2}+$ $2\left|H^{(3)}\right|^{2}$ which are the squares of the components $H_{a_{||} b_{\| \mid} c_{\perp}}$ and $H_{a_{\| \mid} b_{||} c_{\| \mid}}$. These terms are then absent in the final $\tilde{\mathcal{R}}_{4}$ expression (4.21) and (4.29). The only change that impacts our conclusion, (4.36), is that the curvature terms $2 \mathcal{R}_{\|}+2 \mathcal{R}_{\|}^{\perp}$ should be replaced by

$$
2 \mathcal{R}_{\|}+2 \mathcal{R}_{\|}^{\perp}-\left|H^{(2)}\right|^{2}-2\left|H^{(3)}\right|^{2} .
$$

As in (4.36), this combination gets bounded by two inequalities, in order to get classical de Sitter solutions for parallel $p=4,5,6$ sources. While this change modifies the final expression, it has little impact on the physics result: we obtain tight constraints on a combination of fields for de Sitter solutions to exist with parallel $p=4,5,6$ sources. The no-go theorems for parallel $p=3,7,8$ sources are not affected at all.

The combination (1) is better motivated than the curvature terms alone, as it now appears to be T-duality invariant, on geometric backgrounds. This statement can be made more precise by considering group manifolds, where the $f_{b c}^{a}$, building the curvature terms, 
are constant, and some are set to zero by the orientifold projection. In addition, the $H$-flux is odd under an orientifold involution, imposing $H^{(3)}=0$ for a constant flux; avoiding the Freed-Witten anomaly also sets $H^{(3)}$ to zero. The (opposite sign of the) combination (1) then reduces to

$$
\begin{aligned}
& \delta^{a b} f^{d_{\|}}{ }_{c_{\|} a_{\|}} f^{c_{\| \mid}} d_{\| \mid} b_{\| \mid}+\frac{1}{2} \delta^{c h} \delta^{d j} \delta_{a b} f^{a_{\|}}{ }_{c_{\| \mid} j_{||}} f^{b_{\| \mid}} h_{\| \mid} d_{||}+\delta^{a b} f^{d_{\perp}}{ }_{c_{\perp} a_{\|}} f^{c_{\perp}}{ }_{d_{\perp} b_{\|}} \\
& +\delta^{a b} \delta^{d g} \delta_{c h} f^{h_{\perp}}{ }_{g_{\perp} a_{\|}} f^{c_{\perp}} d_{\perp} b_{\| \mid}+\frac{1}{2} \delta^{a d} \delta^{b e} \delta^{c f} H_{a_{\|} b_{\| \mid} c_{\perp}} H_{d_{\| e_{\|}} f_{\perp}},
\end{aligned}
$$

and the first and third terms vanish on nilmanifolds. The $H$-flux component schematically transforms under T-duality into one or the other structure constant, depending on the T-duality direction

$$
H_{a_{\| \mid} b_{\|} c_{\perp}} \rightarrow f^{c_{\| \mid}} a_{|| \mid} b_{\| \mid} \text {or }-f^{a_{\perp}} c_{\perp} b_{\|},
$$

showing the T-duality invariance of the combination (1) in that setting.

A practical consequence for the paper is that several occurrences of "curvature terms" should be replaced by the above "field combination": it is the case for equations (1.2), (1.3), (4.30), and the text of the Outlook. The discussed consequences of the results are unchanged: to start with, the remark on the solutions T-dual to one with an $O_{3}$, at the end of section 4.2 , remains valid. The requirement of having $f^{a_{\|} b_{\perp} c_{\perp}} \neq 0$ for a de Sitter solution still holds, from the constraints on the new combination, implying the nogo theorem for $p=8$ (footnote 6 ) and the impossibility to embed a specific monodromy inflation mechanism, as mentioned in the Outlook.

\section{Corrected equations}

For a $p$-dimensional source, any internal flux $F_{q}$ was decomposed in (4.11) as $F_{q}=$ $\sum_{n=0}^{p-3} F_{q}^{(n)}$, where the components of $F_{q}^{(n)}$ have $n$ internal parallel flat indices, and $F_{q}^{(0)}=$ $\left.F_{q}\right|_{\perp}$. As a consequence, one has

$$
\begin{aligned}
\left|F_{q}\right|^{2} & =\sum_{n=0}^{p-3}\left|F_{q}^{(n)}\right|^{2}, \text { where }\left|F_{q}\right|^{2}=\frac{1}{q !} F_{q a_{1} \ldots a_{q}} F_{q}^{a_{1} \ldots a_{q}}, \\
\left|F_{q}^{(n)}\right|^{2} & =\frac{1}{n !(q-n) !} F_{q} a_{1 \| \ldots a_{n \|} \mid a_{n+1 \perp} \ldots a_{q \perp}} F_{q}^{a_{1 \| \mid \ldots} \ldots a_{n||} a_{n+1 \perp} \ldots a_{q \perp}},
\end{aligned}
$$

the indices being lifted by the flat internal metric. We now consider the trace of the Einstein equation along the internal parallel directions. An internal flux $F_{q}$ appears in it as follows

$$
\begin{aligned}
\delta^{a b} \frac{1}{(q-1) !} F_{q} a_{1||} a_{2} \ldots a_{q} F_{q} b_{1 \|} a_{2} \ldots a_{q} & =\sum_{n \geq 1}^{p-3} \delta^{a b} \frac{1}{(n-1) !(q-n) !} F_{q \quad a_{1||} a_{2 \|} \ldots a_{n \|} a_{n+1 \perp \ldots} \ldots a_{q \perp}}^{(n)} \\
& \times F_{q}^{(n)} b_{1 \|} a_{2||} \ldots a_{n \|} a_{n+1 \perp} \ldots a_{q \perp} \\
= & \sum_{n \geq 0}^{p-3} n\left|F_{q}^{(n)}\right|^{2}=\left|F_{q}\right|^{2}-\left.\left|F_{q}\right|_{\perp}\right|^{2}+\sum_{n \geq 2}^{p-3}(n-1)\left|F_{q}^{(n)}\right|^{2} .
\end{aligned}
$$


The last sum is absent of (4.14) and (4.15). These two equations are corrected towards

$$
\begin{aligned}
\mathcal{R}_{6 \|}+2(\nabla \partial \phi)_{6 \| \mid}= & \frac{p-3}{4}\left(\mathcal{R}_{4}+2(\nabla \partial \phi)_{4}+2 e^{2 \phi}\left|F_{6}\right|^{2}\right) \\
& +\frac{1}{2}\left(|H|^{2}-\left.|H|_{\perp}\right|^{2}+e^{2 \phi}\left(\left|F_{2}\right|^{2}-\left.\left|F_{2}\right|_{\perp}\right|^{2}+\left|F_{4}\right|^{2}-\left.\left|F_{4}\right|_{\perp}\right|^{2}\right)\right. \\
& +\frac{1}{2} \sum_{n \geq 2}^{p-3}(n-1)\left(\left|H^{(n)}\right|^{2}+e^{2 \phi}\left(\left|F_{2}^{(n)}\right|^{2}+\left|F_{4}^{(n)}\right|^{2}\right)\right) \\
\mathcal{R}_{6 \| \mid}+2(\nabla \partial \phi)_{6 \| \mid}= & \frac{p-3}{4}\left(\mathcal{R}_{4}+2(\nabla \partial \phi)_{4}+e^{2 \phi}\left|F_{5}\right|^{2}\right) \\
& +\frac{1}{2}\left(|H|^{2}-\left.|H|_{\perp}\right|^{2}+e^{2 \phi}\left(\left|F_{1}\right|^{2}-\left.\left|F_{1}\right|_{\perp}\right|^{2}+\left|F_{3}\right|^{2}-\left.\left|F_{3}\right|_{\perp}\right|^{2}\right)\right. \\
& +\frac{1}{4} e^{2 \phi}\left(\left|F_{5}\right|^{2}-\left.\left|F_{5}\right|_{\perp}\right|^{2}-\left|*_{6} F_{5}\right|^{2}+\left.\left|\left(*_{6} F_{5}\right)\right|_{\perp}\right|^{2}\right) \\
& +\frac{1}{2} \sum_{n \geq 2}^{p-3}(n-1)\left(\left|H^{(n)}\right|^{2}+e^{2 \phi}\left(\left|F_{3}^{(n)}\right|^{2}+\frac{1}{2}\left|F_{5}^{(n)}\right|^{2}\right)\right),
\end{aligned}
$$

where in IIB, the one-form fluxes, $F_{1}$ and $*_{6} F_{5}$, do not contribute to the new terms because the sum starts with $n \geq 2$. For the same reason, these new terms only contribute for $p \geq 5$. A general rewriting of these two equations, correcting equation (4.16), is then given by

$$
\begin{aligned}
2 \mathcal{R}_{6||}+ & 4(\nabla \partial \phi)_{6||}-\frac{p-3}{2}\left(\mathcal{R}_{4}+2(\nabla \partial \phi)_{4}\right)=|H|^{2}-\left.|H|_{\perp}\right|^{2}+e^{2 \phi}\left(\left|F_{k-2}\right|^{2}-\left.\left|F_{k-2}\right|_{\perp}\right|^{2}\right) \\
& +e^{2 \phi}\left(\left|F_{k}\right|^{2}-\left.\left|F_{k}\right|_{\perp}\right|^{2}+\left|F_{k+2}\right|^{2}+(9-p)\left|F_{k+4}\right|^{2}+5\left|F_{k+6}\right|^{2}+\frac{1}{2}\left(\left.\left|\left(*_{6} F_{5}\right)\right|_{\perp}\right|^{2}-\left.\left|F_{5}\right|_{\perp}\right|^{2}\right)\right) \\
& +\sum_{n \geq 2}^{p-3}(n-1)\left(\left|H^{(n)}\right|^{2}+e^{2 \phi}\left(\left|F_{k}^{(n)}\right|^{2}+\left|F_{k+2}^{(n)}\right|^{2}+\frac{p-6}{2}\left|F_{k+4}^{(n)}\right|^{2}+\frac{p-7}{4}\left|F_{5}^{(n)}\right|^{2}\right)\right),
\end{aligned}
$$

where the $F_{5}$ terms should only be considered in IIB. Equation (4.17) gets corrected by adding the same new line, while the final formula (4.21) becomes

$$
\begin{aligned}
& 2 e^{-2 A} \tilde{\mathcal{R}}_{4}=-\left|*_{\perp} H\right|_{\perp}+\left.\left.\varepsilon_{p} e^{\phi} F_{k-2}\right|_{\perp}\right|^{2}-2 e^{2 \phi}\left|g_{s}^{-1} \tilde{*}_{\perp} \mathrm{d} e^{-4 A}-\varepsilon_{p} F_{k}^{(0)}\right|^{2} \\
& -\sum_{a_{\|}}\left|*_{\perp}\left(\mathrm{d} e^{a_{\|}}\right)\right|_{\perp}-\left.\varepsilon_{p} e^{\phi}\left(\iota_{a_{\| \mid}} F_{k}^{(1)}\right)\right|^{2}-2 \mathcal{R}_{\|}-2 \mathcal{R}_{\|}^{\perp} \\
& -2 e^{-2 A}\left(\mathrm{~d}\left(e^{8 A_{\tilde{*}} \perp} \mathrm{d} e^{-4 A}-e^{8 A} \varepsilon_{p} g_{s} F_{k}^{(0)}\right)\right)_{\widetilde{\perp}} \\
& -e^{2 \phi}\left(\left|F_{k}\right|^{2}-\left|F_{k}^{(0)}\right|^{2}-\left|F_{k}^{(1)}\right|^{2}+2\left|F_{k+2}\right|^{2}+(p-5)\left|F_{k+4}\right|^{2}+\frac{1}{2}\left(\left.\left|F_{5}\right|_{\perp}\right|^{2}-\left.\left|\left(*_{6} F_{5}\right)\right|_{\perp}\right|^{2}\right)\right) \\
& +\sum_{n \geq 2}^{p-3}(n-1)\left(\left|H^{(n)}\right|^{2}+e^{2 \phi}\left(\left|F_{k}^{(n)}\right|^{2}+\left|F_{k+2}^{(n)}\right|^{2}+\frac{p-6}{2}\left|F_{k+4}^{(n)}\right|^{2}+\frac{p-7}{4}\left|F_{5}^{(n)}\right|^{2}\right)\right) \text {. }
\end{aligned}
$$

We now detail the last two lines of (8): they are equal to

$$
\begin{aligned}
& p=3: 0 \\
& p=4:-2 e^{2 \phi}\left|F_{6}\right|^{2}
\end{aligned}
$$




$$
\begin{aligned}
& p=5:\left|H^{(2)}\right|^{2}-e^{2 \phi}\left(2\left|F_{5}\right|^{2}-\left.\frac{1}{2}\left|\left(*_{6} F_{5}\right)\right|_{\perp}\right|^{2}-\frac{1}{2}\left|F_{5}^{(2)}\right|^{2}\right) \\
& p=6:\left|H^{(2)}\right|^{2}+2\left|H^{(3)}\right|^{2}-e^{2 \phi}\left(2\left|F_{4}\right|^{2}-\left|F_{4}^{(2)}\right|^{2}-2\left|F_{4}^{(3)}\right|^{2}+\left|F_{6}\right|^{2}\right) \\
& p=7:\left|H^{(2)}\right|^{2}+2\left|H^{(3)}\right|^{2}-e^{2 \phi}\left(2\left|F_{3}\right|^{2}-\left|F_{3}^{(2)}\right|^{2}-2\left|F_{3}^{(3)}\right|^{2}+2\left|F_{5}\right|^{2}-\left.\frac{1}{2}\left|\left(*_{6} F_{5}\right)\right|_{\perp}\right|^{2}\right. \\
& \left.-\frac{1}{2} \sum_{n \geq 2}^{4}(n-1)\left|F_{5}^{(n)}\right|^{2}\right) \\
& p=8:\left|H^{(2)}\right|^{2}+2\left|H^{(3)}\right|^{2}-e^{2 \phi}\left(2\left|F_{2}\right|^{2}-\left|F_{2}^{(2)}\right|^{2}+3\left|F_{4}\right|^{2}-\sum_{n \geq 2}^{4}(n-1)\left|F_{4}^{(n)}\right|^{2}\right) .
\end{aligned}
$$

We used (4), that leads to the cancelation of all $F_{k}$ terms. That equation, together with $\left|F_{5}\right|^{2}=\left|*_{6} F_{5}\right|^{2} \geq\left.\left|\left(*_{6} F_{5}\right)\right|_{\perp}\right|^{2}$, allows us to prove that the Ramond-Ramond contributions to these lines are always negative (semi-)definite. We rewrite the final equation (8) as

$$
\begin{aligned}
& 2 e^{-2 A} \tilde{\mathcal{R}}_{4}=-\left|*_{\perp} H\right|_{\perp}+\left.\left.\varepsilon_{p} e^{\phi} F_{k-2}\right|_{\perp}\right|^{2}-2 e^{2 \phi}\left|g_{s}^{-1} \tilde{\mathcal{*}}_{\perp} \mathrm{d} e^{-4 A}-\varepsilon_{p} F_{k}^{(0)}\right|^{2} \\
& -\sum_{a_{\|}}\left|*_{\perp}\left(\mathrm{d} e^{a_{\|}}\right)\right|_{\perp}-\left.\varepsilon_{p} e^{\phi}\left(\iota_{a_{\| \mid}} F_{k}^{(1)}\right)\right|^{2}-2 \mathcal{R}_{\|}-2 \mathcal{R}_{\|}^{\perp}+\left|H^{(2)}\right|^{2}+2\left|H^{(3)}\right|^{2} \\
& -2 e^{-2 A}\left(\mathrm{~d}\left(e^{8 A_{\tilde{*} \perp}} \mathrm{d} e^{-4 A}-e^{8 A} \varepsilon_{p} g_{s} F_{k}^{(0)}\right)\right)_{\tilde{\perp}} \\
& -e^{2 \phi}\left(2\left|F_{k+2}\right|^{2}+(p-5)\left|F_{k+4}\right|^{2}+\frac{1}{2}\left(\left.\left|F_{5}\right|_{\perp}\right|^{2}-\left.\left|\left(*_{6} F_{5}\right)\right|_{\perp}\right|^{2}\right)\right. \\
& \left.-\sum_{n \geq 2}^{p-3}(n-1)\left(\left|F_{k+2}^{(n)}\right|^{2}+\frac{p-6}{2}\left|F_{k+4}^{(n)}\right|^{2}+\frac{p-7}{4}\left|F_{5}^{(n)}\right|^{2}\right)\right),
\end{aligned}
$$

where the last two lines are a negative (semi-)definite contribution. The new combination (1) now appears. The integral version of this expression, (4.29), is similarly corrected. Turning to the no-go theorems, equations (4.33), (4.34) and (4.35) still hold in view of (7), the corrected version of (4.16). They can however be refined with the new $H$-flux terms, towards

$$
2 \mathcal{R}_{6 \| \mid}+4(\nabla \partial \phi)_{6 \|}-\frac{p-3}{2}\left(\mathcal{R}_{4}+2(\nabla \partial \phi)_{4}\right)-\left|H^{(2)}\right|^{2}-2\left|H^{(3)}\right|^{2} \geq 0,
$$

for (4.33). We deduce the following version of the main result, correcting (4.36)

$$
\begin{aligned}
& \text { There is no de Sitter vacuum for } p=4,5 \text {, or } 6 \text {, if the inequalities } \\
& -\left.\int_{\widetilde{\mathcal{M}}} \widetilde{\operatorname{vol}}_{6} e^{2 A} \sum_{a_{\|}}\left|\left(\mathrm{d}^{a_{\|} \mid}\right)\right|_{\perp}\right|^{2}<\int_{\widetilde{\mathcal{M}}} \widetilde{\operatorname{vol}}_{6} e^{2 A}\left(2 \mathcal{R}_{\|}+2 \mathcal{R}_{\|}^{\perp}-\left|H^{(2)}\right|^{2}-2\left|H^{(3)}\right|^{2}\right)<0 \\
& \text { are not satisfied. }
\end{aligned}
$$

Open Access. This article is distributed under the terms of the Creative Commons Attribution License (CC-BY 4.0), which permits any use, distribution and reproduction in any medium, provided the original author(s) and source are credited. 